\title{
Establishment and Validation of the Detection of TERT Promoter Mutations by Human Gliomas U251 Cell Lines
}

\author{
Huili Bai, ${ }^{1}$ Shunjie Bai $\mathbb{D}^{2}{ }^{2}$ Xiaosong Li, ${ }^{1}$ Yangli Zhang, ${ }^{1}$ Ying $\mathrm{Li}^{1}{ }^{1}$ Fang He $\mathbb{D},{ }^{3}$ \\ and Wei Cheng ${ }^{1}{ }^{1}$ \\ ${ }^{1}$ The Center for Clinical Molecular Medical Detection, The First Affiliated Hospital of Chongqing Medical University, \\ 1 Friendship Road, Yuzhong District, Chongqing 400016, China \\ ${ }^{2}$ Department of Clinical Laboratory, The First Affiliated Hospital of Chongqing Medical University, 1 Friendship Road, \\ Yuzhong District, Chongqing 400016, China \\ ${ }^{3}$ Department of pharmacy, The First Affiliated Hospital of Chongqing Medical University, 1 Friendship Road, Yuzhong District, \\ Chongqing 400016, China
}

Correspondence should be addressed to Shunjie Bai; jasenbai@cqmu.edu.cn, Fang He; zhangyuhong0730@126.com, and Wei Cheng; chengwei@hospital.cqmu.edu.cn

Received 23 April 2020; Revised 22 July 2020; Accepted 13 August 2020; Published 1 June 2021

Academic Editor: Zhirong Sun

Copyright (@) 2021 Huili Bai et al. This is an open access article distributed under the Creative Commons Attribution License, which permits unrestricted use, distribution, and reproduction in any medium, provided the original work is properly cited.

\begin{abstract}
Gliomas are the most common type of primary brain tumor, yet the prognosis for glioma patients remains poor. Mutations in the promoter region of the telomerase reverse transcriptase gene (TERTp) are associated with diagnosis and poor prognosis in gliomas. Here, we developed a precise and rapid Sanger sequencing assay to screen or TERTp mutations. We established the Sanger sequencing approach for the detection of TERTp mutations based on human glioma cell lines U251 and assessed the analytical validation by determining the accuracy, sensitivity, precision, and specificity. In our study, we verified the accuracy of Sanger sequencing by the real-time polymerase chain reaction method. Our data showed that TERTp mutations were detected at an analytical sensitivity of $10 \%$ per mutant. The precision and specificity validation also showed the desired results. In total, 147 glioma patients were investigated for TERTp mutations, and of each patient, clinical data and molecular characteristics were analyzed. We found that anaplastic oligodendroglioma had the highest frequency of TERTp mutations (66.7\%). No differences in TERTp mutation frequency were observed between frozen tissue specimens and formalin-fixed and paraffin-embedded tissue. TERTp mutations were associated with older patients ( $\geq 45$ years), whereas isocitrate dehydrogenase (IDH) mutations were inclined to a younger age $(<45$ years), frontal location, and pathologic stage II-III patients. IDH mutations were significantly associated with O6-methylguanineDNA methyltransferase $($ MGMT) methylation $(P=0.003)$ and lower Ki-67 protein expression $(P=0.011)$. Moreover, MGMT methylation was enriched in IDH-mutant/TERTp-mutant gliomas, and Ki-67 protein expression was the highest in the IDH-wild type/TERTp-mutant group. Taken together, the findings of this study indicate the establishment of a rapid, precise, and practical Sanger sequencing technique for TERTp mutations in gliomas that may show promising results in clinical applications.
\end{abstract}

\section{Introduction}

Gliomas are one of the most frequent types of primary malignant tumors in the central nervous system (CNS), accounting for $75 \%$ of all primary malignant brain tumors [1-4]. The highly invasive ability of these tumors hampers curative surgical resection and makes disease recurrence ineluctable [5]. Therefore, accurate diagnosis in glioma subgroups is of utmost importance for proper management of patients who suffer from this disease [6]. In a rapidly developing era, the diagnosis of gliomas is not only based on histopathology but also based on additional biomolecular makers, such as $1 \mathrm{p} / 19 \mathrm{q}$ codeletion and isocitrate dehydrogenase (IDH) $1 / 2$ mutations $[7,8]$. Nonetheless, the 5 -year prognosis of gliomas patients is still poor [9]. Thus, it is essential to identify novel biomolecular makers involved in malignant 
transformation to improve diagnosis, prognosis, and treatment responses.

Recently, dysfunction of telomerase reverse transcriptase (TERT), the catalytic subunit of telomerase, has been demonstrated to involve in telomere maintenance and cell proliferation, contributing to human disease progression such as melanomas [10], non-small-cell lung cancer [11], bladder cancer [12], hepatocellular carcinomas [13], thyroid cancer [14], and brain tumors $[15,16]$. In previous studies, the mutations in the promoter region of telomerase reverse transcriptase (TERTp) were associated with tumor biology [17]. Intriguingly, TERTp mutations affected survival and disease recurrence in bladder cancer [12]. Moreover, TERTp mutations play important roles in several health problems such as gliomas [18]. Based on the 2016 World Health Organization (WHO) classification of CNS tumors, TERTp mutations have been incorporated into glioma diagnosis [19]. Telomerase activity plays a pivotal role in tumorigenesis and facilitates the development of different brain tumors, including astrocytomas, primary glioblastomas (GBMs), and oligodendrogliomas [20, 21]. The two most frequent mutations (chr5: 1,295, $228 \mathrm{C}>\mathrm{T}$ and 1,295, $250 \mathrm{C}>\mathrm{T}$, respectively), located at $-124 \mathrm{bp}$ and $-146 \mathrm{bp}$, independently generate an identical $11 \mathrm{bp}$ nucleotide sequence containing a consensus binding site for E-twenty-six (ETS) transcription factors and have been associated with increased mRNA expression or TERTp transcriptional activity [22]. Knockdown of human TERT inhibited cell proliferation and migration of gliomas in vivo [23]. Hence, detecting TERTp mutations in a clinical setting is critical.

Current techniques, such as Amplification Refractory Mutation System PCR (ARMS-PCR) and droplet digital PCR (ddPCR) for mutation detection, are limited in terms of screening unknown mutations. Furthermore, next-generation sequencing (NGS) often takes a while to run, analyzing data is time-consuming, and it can be costly and of high requirement. Sanger sequencing is a widely used technique to interrogate genes for small fragment mutations and acts as a gold standard for mutation detection. In this study, we aimed to develop a Sanger sequencing assay to achieve the precise and rapid detection of the TERTp mutations in gliomas and simultaneously assessed analytical validation and clinical utility.

\section{Materials and Methods}

2.1. Clinical Samples. Glioma samples were collected from 147 patients who underwent surgery at The Department of Neurosurgery, the First Affiliated Hospital of Chongqing Medical University (Chongqing, China), between June 2016 and April 2019. Among the tumors were 37 astrocytomas (DA), 15 anaplastic astrocytomas (AA), 24 anaplastic oligodendrogliomas (AO), 6 oligoastrocytomas (OA), 28 oligodendrogliomas (O), and 37 GBMs. Patients were diagnosed in accordance with pathological diagnosis. Clinicopathological data, including sex, age, tumor location, histologic type, pathologic stage, and Ki-67 protein expression, were retrospectively retrieved from respective institutional medical record systems. Formalin-fixed paraffin-embedded (FFPE) tumor tissues $(47,31.97 \%)$ and frozen tissues (100, 68.03\%) of 147 glioma cases underwent molecular detection. Our study was approved by the ethics committee of the First Affiliated Hospital of Chongqing Medical University (Chongqing, China). Written informed consent (no. 2020-420), including molecular genetic testing and medical record reviews, was obtained from each patient or their next of kin. Data were anonymized prior to analysis to protect the patients' identify.

2.2. Cell Lines. Two human glioma cell lines, including U251 and OL, were kindly provided by the Chongqing Key Laboratory of Neurobiology (Chongqing Medical University). Cell lines were maintained in high-glucose Dulbecco's modified Eagle's medium (DMEM, HyClone, USA) supplemented with $10 \%$ fetal bovine serum (FBS, Gibco, USA) and $1 \%$ penicillin/streptomycin at $37^{\circ} \mathrm{C}$ in $5 \% \mathrm{CO}_{2}$. Genomic DNA from both two cell lines was extracted for analytical validation.

2.3. DNA Extraction. For FFPE samples, six $5 \mu \mathrm{m}$ thick sections were cut from each selected block, followed by one section that was H\&E-stained. The tumor area was marked on the control slide, and the tumor cell purity was more than $50 \%$. DNA was extracted from the tumor-rich areas using a universal FFPE DNA extraction kit (Amoy Diagnostics Co. Ltd., Xiamen, China). Part of the DNA samples was isolated from frozen sections as previously described [24]. Genomic DNA was extracted from human glioma cell lines using a commercial kit (Tiangen Biotech Co., Ltd., Beijing, China). The DNA concentration was quantified using a Qubit 3.0 fluorometer (Invitrogen, Carlsbad, CA, USA).

2.4. Sequencing of TERTp and IDH1/2 Mutations. DNA ( $4 \mathrm{ng}$ ) was amplified by PCR using specific primers presented in Table 1. The M13F and M13R primers used are a pair of universal sequence-tagged primers, and M13 primers were used as sequencing primers (Table 1 ). The PCR reaction was performed using the following program: initial denaturation at $95^{\circ} \mathrm{C}$ for $10 \mathrm{~min}$, followed by 30 cycles at $95^{\circ} \mathrm{C}$ for $30 \mathrm{sec}$, primer annealing at $62^{\circ} \mathrm{C}$ for $30 \mathrm{sec}$, and $72^{\circ} \mathrm{C}$ for $1 \mathrm{~min}$, final extension took place at $72^{\circ} \mathrm{C}$ for $7 \mathrm{~min}$. Amplification PCR and PCR sequencing were performed using the BigDye ${ }^{\circledR}$ Direct Cycle Sequencing Kit (Applied Biosystems, Foster City, CA, USA) according to the manufacturer's guidelines. The DNA sequencing products were purified using the BigDye ${ }^{\circledR}$ XTerminator ${ }^{\mathrm{TM}}$ Purifcation Kit (Applied Biosystems, Foster City, CA, USA), then resolved by capillary electrophoresis on a 3500DX Genetic Analyser (Applied Biosystems, Foster City, CA, USA). The entire experiment took approximately 4 hours.

2.5. RT-PCR (Real-Time Polymerase Chain Reaction) for TERTp Mutations. TERTp mutations were screened by RTPCR. The PCR reagent and probe were from a commercial kit (Genetron Health Co. Ltd., Beijing, China). PCR was carried out in four stages: activation the Uracil-DNA Glycosylase (UNG) at $37^{\circ} \mathrm{C}$ for $3 \mathrm{~min}$; incubation at $95^{\circ} \mathrm{C}$ for $3 \mathrm{~min}$ to activate DNA polymerase; enrichment of the mutant allele for $15 \mathrm{cycles}$ at $95^{\circ} \mathrm{C}$ for $15 \mathrm{sec}$ and $65^{\circ} \mathrm{C}$ for $45 \mathrm{sec}$; and TaqMan PCR for 25 cycles at $95^{\circ} \mathrm{C}$ for $15 \mathrm{sec}$ and $60^{\circ} \mathrm{C}$ for $45 \mathrm{sec}$ (fluorescence collection). Target genes and the internal control were labeled with FAM and ROX probes, respectively. 
TABle 1: Primer sequences per gene.

\begin{tabular}{lccc}
\hline Genes & Forward primer $\left(5^{\prime}-3^{\prime}\right)$ & Reverse primer $\left(5^{\prime}-3^{\prime}\right)$ & Amplified fragments \\
\hline TERT & M13F-AGTGGATTCGCGGGCACAGA & M13R-CAGCGCTGCCTGAAACTC & 235 bp \\
IDH1 & M13F-GAGAAGAGGGTTGAGGAGTT & M13R-TACCTTGCTTAATGGGTGTA & 294 bp \\
IDH2 & M13F-GCTTGGGGTTCAAATTCTG & M13R-CGGTCTGCCACAAAGTCTG & 343 bp \\
M13-tagged primer & TGTAAAACGACGGCCAGT & CAGGAAACAGCTATGACC & $/$ \\
MSP-M & TTTCGACGTTCGTAGGTTTTCGC & GCACTCTTCCGAAAACGAAACG & 81 bp \\
MSP-U & TTTGTGTTTGATGTTTGTAGGTTTT & AACTCCACACTCTTCCAAAAACAAAA & 93 bp \\
\hline
\end{tabular}

TERT: telomerase reverse transcriptase; IDH1: isocitrate dehydrogenase 1; IDH2: isocitrate dehydrogenase 2; M13F: M13 universal forward sequence-tagged primers; M13R: M13 universal reverse sequence-tagged primers. MSP-M: methylation special PCR methylated primers; MSP-U: methylation special PCR unmethylated primers.

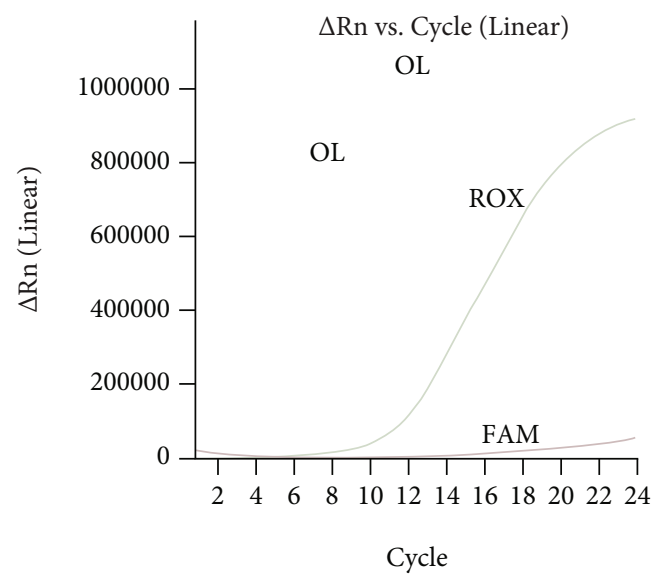

(a)

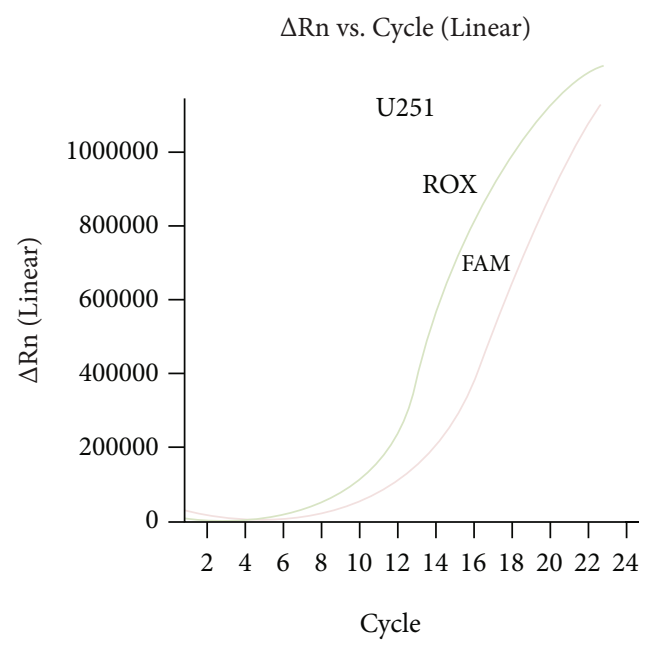

(c)

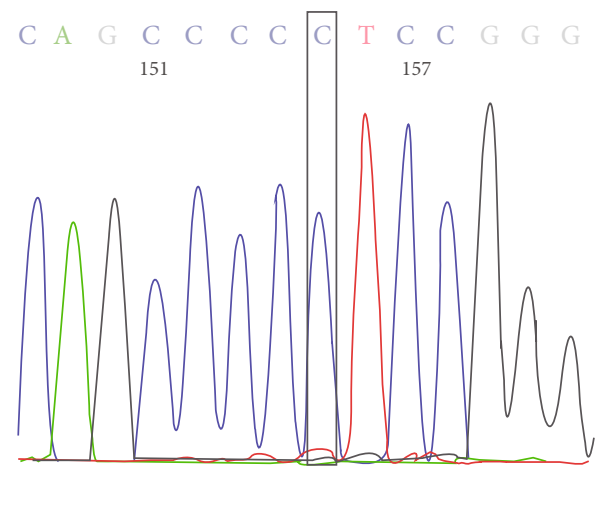

(b)

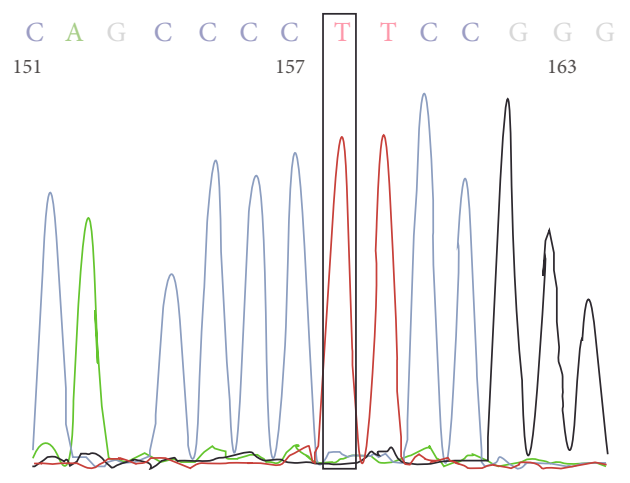

(d)

FIgURE 1: Accuracy of the Sanger sequencing assay for TERTp mutations in comparison with the RT-PCR method. Genomic DNA from OL cell lines was used for the detection of TERTp mutations by the RT-PCR method (a) and Sanger sequencing assays (b). OL cell lines presented wild-type TERTp. Genomic DNA from the U251 cell line was used for the detection of TERTp mutations by the RT-PCR method (c) and Sanger sequencing assays (d). U251 cell lines harbored C228T homozygous mutation of the TERTp. The TERTp target gene and internal control were labeled with FAM and ROX probes, respectively. TERTp: the promoter region of the telomerase reverse transcriptase gene.

Reactions were carried out in single-sealed tubes and detected using a 7500 Real-Time PCR instrument (Applied Biosystems, Foster City, CA, USA).
2.6. Methylation-Specific PCR (MSP). The methylation status of the MGMT gene promoter was detected by extracting tumor DNA for bisulfite conversion using the EZ DNA 


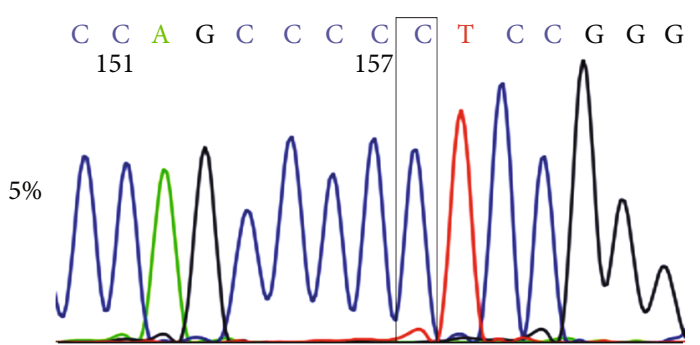

(a)

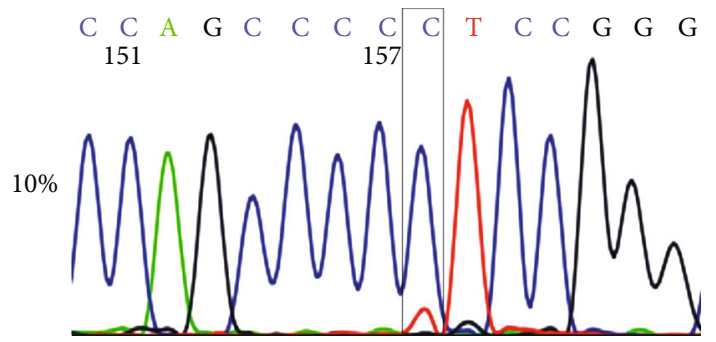

(c)

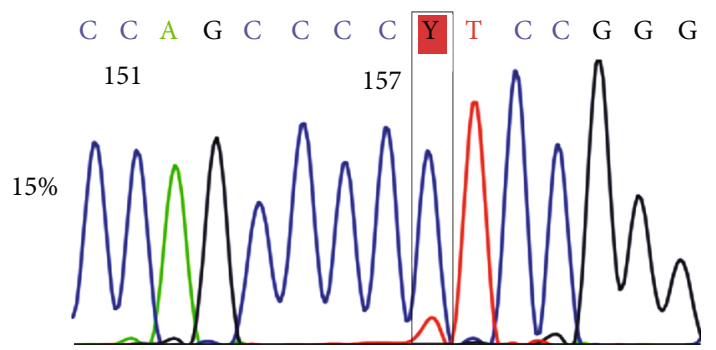

(e)

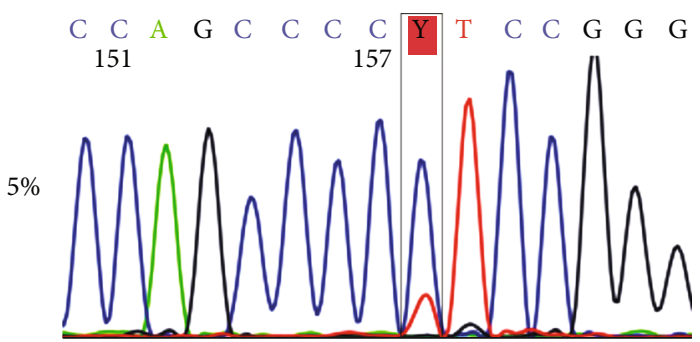

(b)

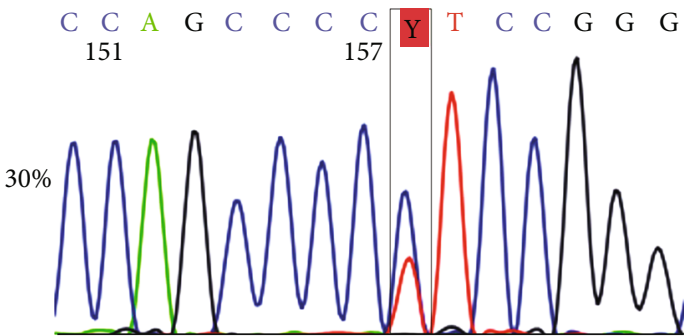

(d)

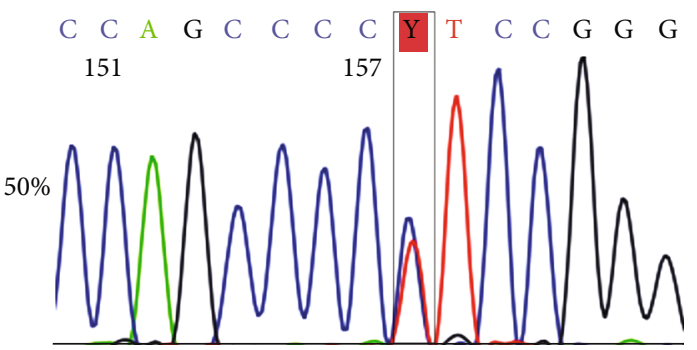

(f)

FIGURE 2: Sensitivity of the Sanger sequencing assay for the TERTp mutation. The percentage of C228T mutants in genomic DNA from mutant and wild-type cell lines (U251: C228T; OL: WT) is 5\% (a), 10\% (b), 15\% (c), 20\% (d), 30\% (e), and 50\% (f). TERTp: the promoter region of the telomerase reverse transcriptase gene.

TABLE 2: Precision of the Sanger sequencing assay for TERTp mutations. Performance of interassay and intra-assay coefficients of variation.

\begin{tabular}{lcccccccccc}
\hline \multirow{2}{*}{ Concentration } & \multirow{2}{*}{} & \multicolumn{3}{c}{ Total } & \multicolumn{3}{c}{ Within-day } & \multicolumn{3}{c}{ Between-day } \\
& & Mean & SD & CV (\%) & Mean & SD & CV (\%) & Mean & SD & CV (\%) \\
\hline $50 \%$ & 30 & 1.14 & 0.12 & 10.72 & 1.21 & 0.02 & 1.84 & 1.20 & 0.12 & 10.00 \\
$15 \%$ & 30 & 7.14 & 0.93 & 13.02 & 5.67 & 0.13 & 2.26 & 6.61 & 0.71 & 10.73 \\
\hline
\end{tabular}

TERTp: the promoter region of the telomerase reverse transcriptase gene; SD: standard deviation; CV: coefficient of variation.

methylation-Gold Kit (Zymo Research, Orange County, CA, USA). Two separate MSP reactions were performed, one using primers specific for methylated MGMT promoter sequences and the other using PCR primers specific for unmethylated MGMT promoter sequences (Table 1) [25].

2.7. Statistical Analysis. GraphPad Prism 5 (La Jolla, CA, USA) was used for all statistical analysis (the Pearson chisquare test or the 2-tailed Fisher's exact test). Statistical significance was indicated as $P<0.05$. Sequence homogeneity was confirmed by comparison of the available sequences on the NCBI's BLAST (basic local alignment search tool) and Chromas software. Cycle threshold (Ct) values [26] were recorded, and corresponding $\Delta \mathrm{Ct}$ values (Ct (TERT target gene assay)-Ct (internal control assay) were calculated. Analytical validation experiments were performed in triplicate.

\section{Results}

3.1. The Establishment of Sanger Sequencing-Based Methods to Detect TERTp Mutations. All samples were successfully analyzed by Sanger sequencing. The contiguous read length of the sequence product was approximately $233 \mathrm{bp}$, and no dimer structure and nonspecific amplifications were observed, which was in line with the primer design software database. Based on the sequence analysis by BLAST, the output sequence showed $100 \%$ homology and perfectly matched with the TERT gene promoter region (data not shown). The reportable range also included the wild-type, C228T, and 


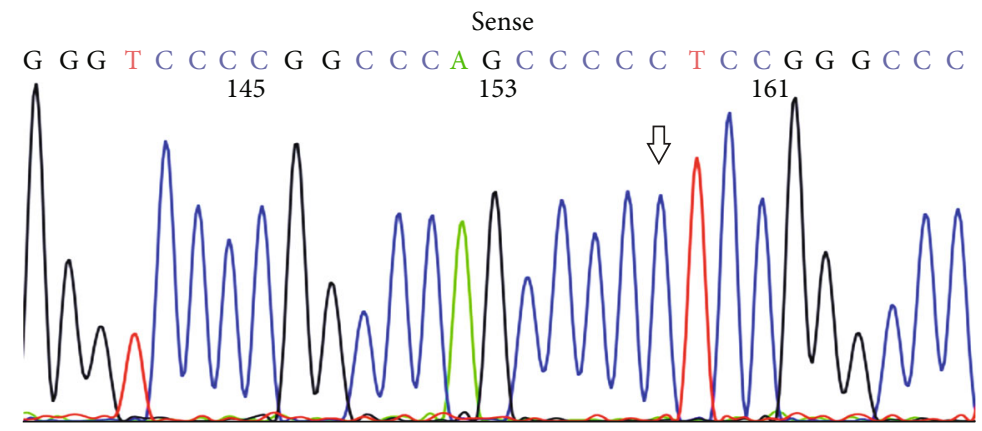

(a)

Anti-sense

G G G C C C G G A G G G G C T G G G C C G G G G A C C C

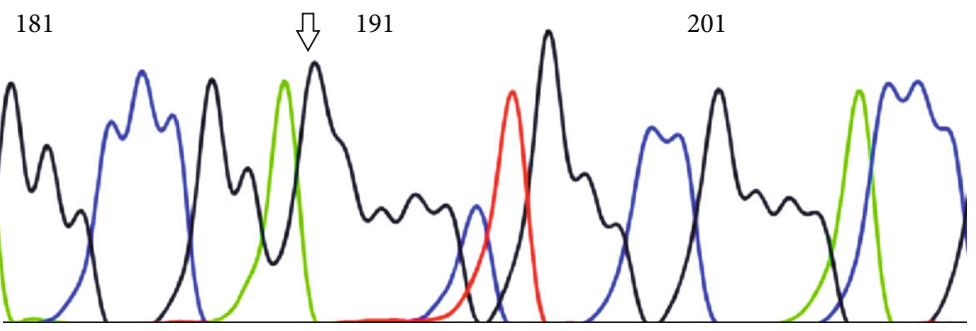

(b)

FIGURE 3: Specificity of the Sanger sequencing assay for TERTp mutations. Shown are representative sequences of wild-type TERTp. (a) Wildtype TERTp as detected by sense sequences. (b) Wild-type TERTp as detected by antisense sequences. Note that the arrowhead points to the nucleotide C228 site of TERTp. TERTp: the promoter region of the telomerase reverse transcriptase gene.

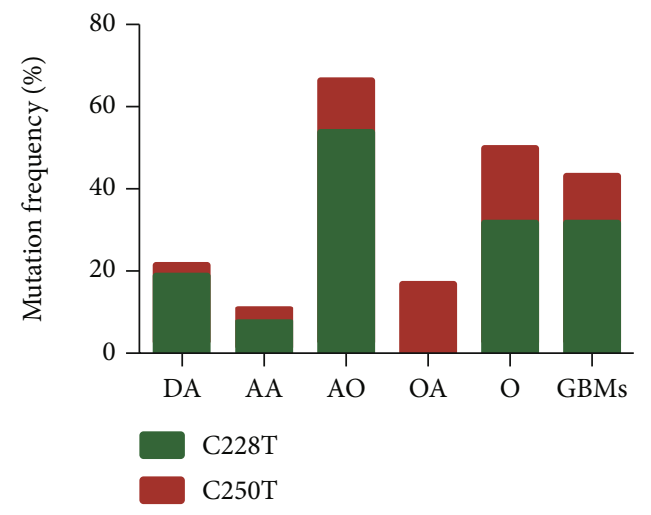

(a)

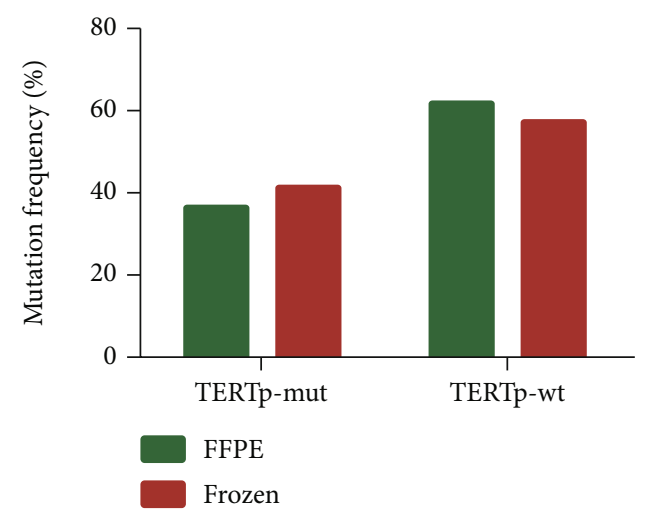

(b)

FIgUre 4: The frequency of TERTp mutations in glioma samples. (a) The frequency of TERTp C228T and C250T mutations in each glioma type (DA, AA, AO, OA, O, and GBMs). (b) The frequency of TERTp mutations in FFPE and frozen tissues. TERTp: the promoter region of the telomerase reverse transcriptase gene. DA: diffuse astrocytoma; AA: anaplastic astrocytoma; AO: anaplastic oligodendroglioma; OA: oligoastrocytoma; O: oligodendroglioma; GBMs: glioblastomas; FFEP: formalin fixed paraffin-embedded; TERTp-mut: TERT promoter mutation; TERTp-wt: TERT promoter wild type.

C250T mutation status, and it was clearly visible and readable. Moreover, the sequencing peak was clear without noise. Figure S1 presents Sanger sequencing data for representative samples.

3.2. Analytical Validation of the TERTp Sequencing Assay. The analytical validation of the TERTp sequencing assay was assessed by determining the accuracy, sensitivity, precision, and specificity.
For the analytical accuracy assay, 20 clinical samples were randomly investigated for TERTp mutations; 12 cases were wild-type, 7 cases contained a C228T mutation, and 1 case contained a C250T mutation. RT-PCR analysis confirmed the sequencing results (Table SI). To further verify the accuracy assay, we analyzed OL and U251 cell lines for TERTp mutations using RT-PCR assay and Sanger sequencing analysis. The results showed that the OL cell line was wild-type for TERTp (Figures 1(a) and 1(b)), 
whereas the U251 cell line harbored a TERTp C228T homozygous mutation (Figures 1(c) and 1(d)).

For the analytical sensitivity assay, we tested the assay on serial dilutions of the C228T target mutation, which were generated by mixing definite ratios of DNA extracted from mutant and wild-type cell lines (U251: C228T; OL: WT) to a panel consisting of $5 \%, 10 \%, 15 \%, 20 \%, 30 \%$, and $50 \%$ of mutants (Figures 2(a)-2(f)). Our data showed that our sequencing method can detect the TERT C228T mutation at a sensitivity of $10 \%$ or more (Figure 2(b)).

For the analytical precision assay, $50 \%$ and $15 \%$ proportion mutation samples were tested for 10 days, respectively (Table 2). The mean, standard deviation (SD), and coefficient of variation $(\mathrm{CV})$ of the $\mathrm{C} 228 \mathrm{~T} / \mathrm{C}$ signal intensity ratio were calculated to assess analytical precision. In this study, the total, within-day, and between-day SD values were 0.12 , 0.02 , and 0.12 for $50 \%$ proportion mutation cases, respectively. The total, within-day, and between-day SD values were $0.93,0.13$, and 0.71 for $15 \%$ proportion mutation cases, respectively. The total, within-day, and between-day CV were $10.72 \%, 1.84 \%$, and $10.00 \%$ for $50 \%$ proportion mutation cases, respectively. The total, within-day, and betweenday CV were $13.02 \%, 2.26 \%$, and $10.73 \%$ for $15 \%$ proportion mutation cases, respectively. Therefore, in the case of both high proportion $(50 \%)$ and low proportion (15\%) mutants, the CV did not exceed $15 \%$, and we concluded that there was evidence of constant precision.

For the analytical specificity assay, the same sample was amplified in duplicate, one for TERTp forward sequencing (Figure 3(a)) and the other for TERTp reverse sequencing (Figure 3(b)). We obtained the same sequence results from forward and reverse sequencing and found a perfect match with the TERTp gene region.

3.3. TERTp Mutations Detected by Sanger Sequencing in Clinical Glioma Samples. TERTp mutations were found in 59 out of 147 gliomas samples (40.13\%). The C228T mutation (44 of 59) was much more frequent than the C250T mutation (15 of 59) in our cohort, and these mutations were mutually exclusive. The frequency of mutants for each histological type is presented in Figure 4(a). The incidence of TERTp mutations was the highest in AO tumor samples (66.7\%), followed by DA (25.0\%), AA (10.8\%), and OA (16.7\%) tumor samples showing a lower incidence, while $\mathrm{O}$ and GBM tumor samples (O, 56.5\%; GBMs, 43.2\%) showed an intermediate incidence when compared with others.

Comparative analyses were performed for the detection of TERTp mutations using a cohort of frozen tissue specimens $(n=100)$ and FFPE samples $(n=47)$. Representative H\&E staining for FFPE and frozen tissues are shown in Figure S2. As shown in Figure 4(b), the median percentage of TERTp mutations from FFPE tissue and frozen tissue was $37.0 \%$ and $42.0 \%$, respectively. No obvious differences were observed between the two tissue specimens.

3.4. Association of TERTp Mutations, IDH Mutations, and MGMT Methylation with Clinicopathological Features from Glioma Patients. Subsequently, we associated TERTp mutations, IDH mutations, and MGMT methylation with clinico-
TABLE 3: Correlations between clinicopathological characteristics and the status of TERTp mutations.

\begin{tabular}{|c|c|c|c|c|}
\hline & \multirow{2}{*}{$\begin{array}{c}\text { Total } \\
n=147(\%)\end{array}$} & \multicolumn{2}{|c|}{ TERTp } & \multirow{2}{*}{$P$ value } \\
\hline & & $\begin{array}{c}\text { Mut } \\
n=59(\%)\end{array}$ & $\begin{array}{c}\mathrm{Wt} \\
n=88(\%)\end{array}$ & \\
\hline Sex & & & & 0.041 \\
\hline Male & $84(57.1)$ & $40(67.8)$ & $44(50.0)$ & \\
\hline Female & $63(42.9)$ & $19(32.2)$ & $44(50.0)$ & \\
\hline Age (years) & & & & $<0.001^{*}$ \\
\hline$<45$ & $70(47.6)$ & $17(28.8)$ & $53(60.2)$ & \\
\hline $45-60$ & $59(40.1)$ & $31(52.5)$ & $28(31.8)$ & \\
\hline$\geq 60$ & $18(12.2)$ & $11(18.6)$ & $7(8.0)$ & \\
\hline Tumor location & & & & 0.729 \\
\hline Frontal & $91(61.9)$ & $38(64.4)$ & $53(60.2)$ & \\
\hline Others & $56(38.1)$ & $21(35.6)$ & $35(39.8)$ & \\
\hline Histology & & & & $0.007^{*}$ \\
\hline DA & $37(25.2)$ & $8(13.6)$ & $29(33.0)$ & \\
\hline AA & $15(10.2)$ & $4(6.8)$ & $11(12.5)$ & \\
\hline $\mathrm{AO}$ & $24(16.3)$ & $16(27.1)$ & $8(9.1)$ & \\
\hline $\mathrm{OA}$ & $6(4.1)$ & $1(1.7)$ & $5(5.7)$ & \\
\hline $\mathrm{O}$ & $28(19.0)$ & $14(23.7)$ & $14(15.9)$ & \\
\hline GBMs & $37(25.2)$ & $16(27.1)$ & $21(23.9)$ & \\
\hline Pathologic stage & & & & 0.338 \\
\hline Grade II-III & $109(74.1)$ & $41(69.5)$ & $68(77.3)$ & \\
\hline Grade IV & $38(25.9)$ & $18(30.5)$ & $20(22.7)$ & \\
\hline Ki-67 & & & & 0.392 \\
\hline$<15 \%$ & $89(60.5)$ & $33(55.9)$ & $56(63.6)$ & \\
\hline$\geq 15 \%$ & $58(39.5)$ & $26(44.1)$ & $32(36.4)$ & \\
\hline IDH gene & & & & 0.312 \\
\hline Mutation & $76(51.7)$ & $34(57.6)$ & $42(47.7)$ & \\
\hline Wild type & $71(48.3)$ & $25(42.4)$ & $46(52.3)$ & \\
\hline MGMT promoter & & & & 0.088 \\
\hline Methylated & $108(73.5)$ & $48(81.4)$ & $60(68.2)$ & \\
\hline Unmethylated & $39(26.5)$ & $11(18.6)$ & $28(31.8)$ & \\
\hline
\end{tabular}

TERTp: the promoter region of the telomerase reverse transcriptase gene; IDH: isocitrate dehydrogenase; MGMT: O6-methylguanine-DNA methyltransferase; Mut: mutation; Wt: wild-type; DA: diffuse astrocytoma; AA: anaplastic astrocytoma; AO: anaplastic oligodendroglioma; $\mathrm{OA}$ : oligoastrocytoma; O: oligodendroglioma; GBMs: glioblastomas. $P$ value was calculated by the Pearson chi-square test or the 2-tailed Fisher's exact test. * Statistically significant: $P<0.05$.

pathological features from glioma patients and pooled analysis was performed, including sex, age, tumor location, histology, pathologic stage, and $\mathrm{Ki}-67$ protein expression. Representative examples of the IDH status, Ki-67 staining, and MGMT status are shown in Figure S3 and S4. As shown in Table 3, TERTp mutations were common in glioma patients over 45 years old $(P<0.001)$. However, no associations were observed with tumor location, pathologic stage, and Ki-67 protein expression. As shown in Table 4, IDH mutations were highly associated with a younger age ( $<45$ years, $P=0.005)$, frontal location $(P<0.001)$, and pathologic stage II-III $(P<0.001)$. IDH mutations were 
TABLE 4: Correlations between clinicopathological characteristics and the status of TERTp mutations.

\begin{tabular}{|c|c|c|c|c|c|c|c|}
\hline & & & & & MGMT & & \\
\hline & $\begin{array}{c}\text { Total } \\
n=147(\%)\end{array}$ & $\begin{array}{c}\text { Mut } \\
n=76(\%)\end{array}$ & $\begin{array}{c}\mathrm{Wt} \\
n=71(\%)\end{array}$ & $P$ value & $\begin{array}{c}M \\
n=108(\%)\end{array}$ & $\begin{array}{c}U \\
n=39(\%)\end{array}$ & $P$ value \\
\hline Sex & & & & 0.505 & & & 0.707 \\
\hline Male & $84(57.1)$ & $41(53.9)$ & $43(60.6)$ & & $63(58.3)$ & $21(53.8)$ & \\
\hline Female & $63(42.9)$ & $35(46.1)$ & $28(39.4)$ & & $45(41.7)$ & $18(46.2)$ & \\
\hline Age (years) & & & & $0.005^{*}$ & & & 0.257 \\
\hline$<45$ & $70(47.6)$ & $42(55.3)$ & $28(39.4)$ & & $50(46.3)$ & $20(51.3)$ & \\
\hline $45-60$ & $59(40.1)$ & $31(40.8)$ & $28(39.4)$ & & $47(43.5)$ & $12(30.8)$ & \\
\hline$\geq 60$ & $18(12.2)$ & $3(3.9)$ & $15(21.1)$ & & $11(10.2)$ & 7 (17.9) & \\
\hline Tumor location & & & & $<0.001^{*}$ & & & $0.022^{*}$ \\
\hline Frontal & $91(61.9)$ & $58(76.3)$ & $33(46.5)$ & & $73(67.6)$ & $18(46.2)$ & \\
\hline Others & $56(38.1)$ & $18(23.7)$ & $38(53.5)$ & & $35(32.4)$ & $21(53.8)$ & \\
\hline Histology & & & & $<0.001^{*}$ & & & $0.009^{*}$ \\
\hline DA & $37(25.2)$ & $20(26.3)$ & $17(23.9)$ & & $22(20.4)$ & $15(38.5)$ & \\
\hline AA & $15(10.2)$ & $6(7.9)$ & $9(12.7)$ & & $14(13.0)$ & $1(2.6)$ & \\
\hline $\mathrm{AO}$ & $24(16.3)$ & $16(21.1)$ & $8(11.3)$ & & $20(18.5)$ & $4(10.3)$ & \\
\hline $\mathrm{OA}$ & $6(4.1)$ & $4(5.3)$ & $2(2.8)$ & & $2(1.9)$ & $4(10.3)$ & \\
\hline $\mathrm{O}$ & $28(19.0)$ & $22(28.9)$ & $6(8.5)$ & & $24(22.2)$ & $4(10.3)$ & \\
\hline GBMs & $37(25.2)$ & $7(9.2)$ & $29(40.8)$ & & $26(24.1)$ & $11(28.2)$ & \\
\hline Pathologic stage & & & & $<0.001^{*}$ & & & 0.286 \\
\hline Grade II-III & $109(74.1)$ & $67(88.2)$ & $42(59.2)$ & & $83(76.1)$ & $26(66.7)$ & \\
\hline Grade IV & $38(25.9)$ & $9(11.8)$ & $29(40.8)$ & & $25(22.9)$ & $13(33.3)$ & \\
\hline $\mathrm{Ki}-67$ & & & & $0.011^{*}$ & & & 1.000 \\
\hline$<15 \%$ & $89(60.5)$ & $54(71.1)$ & $35(49.3)$ & & $65(60.2)$ & $24(61.5)$ & \\
\hline$\geq 15 \%$ & $58(39.5)$ & $22(28.9)$ & $36(50.7)$ & & $43(39.8)$ & $15(38.5)$ & \\
\hline MGMT promoter & & & & $0.003^{*}$ & & & - \\
\hline Methylated & $108(73.5)$ & $64(84.2)$ & $44(62.0)$ & & - & - & \\
\hline Unmethylated & $39(26.5)$ & $12(15.8)$ & $27(38.0)$ & & - & - & \\
\hline TERT promoter & & & & 0.312 & & & 0.088 \\
\hline Mutation & $59(40.1)$ & $34(44.7)$ & $25(35.2)$ & & $48(44.4)$ & $11(28.2)$ & \\
\hline Wild type & $88(59.9)$ & $42(55.3)$ & $46(64.8)$ & & $60(55.6)$ & $28(71.8)$ & \\
\hline
\end{tabular}

TERTp: the promoter region of the telomerase reverse transcriptase gene; IDH: isocitrate dehydrogenase; MGMT: O6-methylguanine-DNA methyltransferase; Mut: mutation; Wt: wild-type; M: methylated; U: unmethylated; DA: diffuse astrocytoma; AA: anaplastic astrocytoma; AO: anaplastic oligodendroglioma; OA: oligoastrocytoma; O: oligodendroglioma; GBMs: glioblastomas. $P$ value was calculated by the Pearson chi-square test or the 2-tailed Fisher's exact test. * Statistically significant: $P<0.05$.

more frequent in MGMT methylated cases compared with unmethylated cases $(P=0.003)$. Furthermore, IDH mutations correlated with low Ki-67 protein expression $(<15 \%, P=0.011)$, and MGMT methylation was more frequently detected in frontally located gliomas $(P=0.022)$. It was independent of pathologic stage, TERTp mutations, and $\mathrm{Ki}-67$ protein expression.

3.5. Association of Both TERTp Mutations and IDH Mutations with MGMT Methylation and Ki-67 Protein Expression. Glioma cases were clustered into four groups: IDH-mut/TERTp-mut (positive for both IDH and TERTp mutations), IDH-mut/TERTp-wt (positive IDH mutation, but negative for TERTp mutations), IDH-wt/TERTp-mut (positive TERTp mutations, but negative for IDH muta- tions), and IDH-wt/TERTp-wt (no IDH or TERTp mutations). As shown in Figure 5, MGMT methylation was enriched in IDH-mut tumors, especially in the IDH-mut/TERTp-mut group.

In this study, we clinical information was collected to analyze the expression of Ki-67 protein, which is a maker of tumor proliferation [27]. Our data showed that Ki-67 protein expression was the highest in the IDH-wt/TERTp-mut group, followed by IDH-wt/TERTp-wt, which also showed high levels of $\mathrm{Ki}-67$ protein expression.

\section{Discussion}

In this study, we developed and evaluated a Sanger sequencing assay of TERTp mutations. Our assay was more rapid 


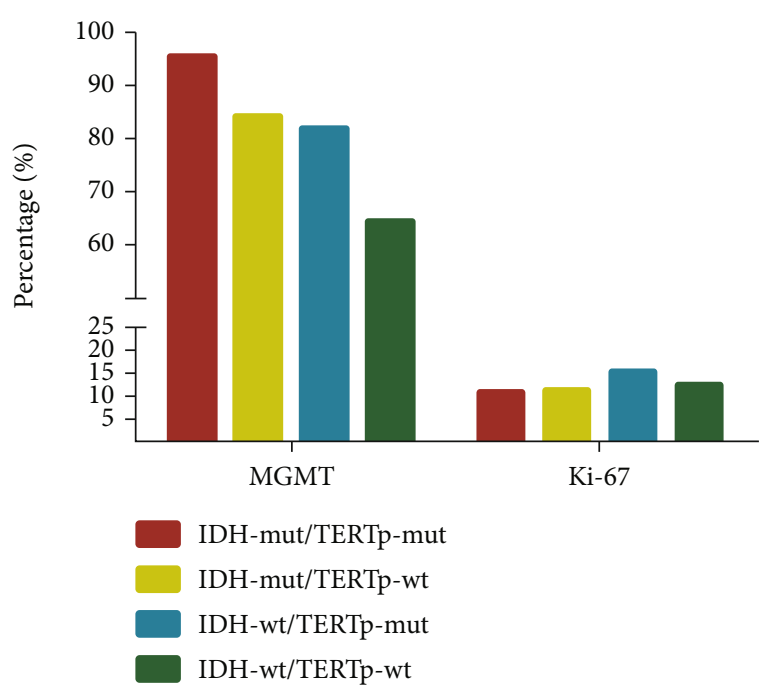

Figure 5: The percentage of MGMT methylation and Ki-67 protein expression in four gliomas subgroups. MGMT methylation was enriched in the IDH-mut/TERTp-mut subgroup, and the percentage of $\mathrm{Ki}-67$ protein expression was the highest in the IDH-wt/TERTp-mut subgroup. MGMT: O6-methylguanine-DNA methyltransferase; TERTp: the promoter region of the telomerase reverse transcriptase gene; IDH: isocitrate dehydrogenase; mut: mutation; wt: wild-type.

than conventional sequencing and can be performed in less than 4 hours. Notably, our method is useful for the $10 \%$ of mutant tumor samples, in which high sensitivity mutation detection is needed for samples with very a low DNA input ( $4 \mathrm{ng}$ ). Clinical tumor tissues are rare and precious. In addition, the use of universal primers may simplify postamplification procedures by allowing all sequencing reactions to be performed with M13F and M13R primers [28]. In previous studies, it was shown that Sanger sequencing lacked the sensitivity for use on glioma samples, which could be prone to low tumor purity and heterogeneity of the gliomas themselves $[28,29]$. To overcome these potential problems, all tumor tissue sections were reevaluated by a professional pathologist to obtain $\geq 50 \%$ higher-purity tumor areas. In some cases, other approaches for mutation detection may have specific advantages. For instance, NGS can simultaneously analyze many molecular alterations with high sensitivity. Other methods, including ARMS-PCR and ddPCR, are highly sensitive and convenient. Moreover, studies have demonstrated that the sensitivity of ARMS-PCR and ddPCR is $0.1 \%$ and $0.01 \%$, respectively [30, 31]. However, whether such a high sensitivity method is required is still controversial as low proportion mutation might be not required for clinical applications.

We found that U251 cells harbored the C228T TERTp mutation and that OL cells did not carry C228T or C250T TERTp mutations. It is particularly important to identify the underlying mechanism for the malignant biological properties of gliomas in vitro study. Indeed, immortalized cell lines can provide strong evidence in the cancer setting [32]. We identified a novel C229T+C228T mutation in a single case of thyroid cancer (data not shown). In addition, the assay may have utility in other genetic mutations, such as IDH1/2 mutations in gliomas [33], and genetic makers can improve clinical targeted therapy development.

In the present study, we performed sequence analysis of TERTp mutations in a cohort of 147 gliomas tumor samples. The results of our study suggested that anaplastic oligodendrogliomas exhibited the highest incidence of TERTp mutations across all glioma types, followed by oligodendroglia. Data were in line with the findings presented in previously published studies $[34,35]$. The strong association between TERTp mutations and the histology of oligodendroglial tumors suggests that TERTp mutations are involved in oncogenesis of oligodendroglial tumors [22]. However, in some studies, it was presented that the highest frequency of TERTp mutations was GBMs [22, 36]. The discrepancy may be explained by population differences, regional differences, or tumor sample size. Most reports on this subject are from Western populations; however, our present study was based on the Han Chinese population. Furthermore, we evaluated FFPE tissue samples and frozen tissue specimens for TERTp mutations. The frequency of FFPE specimens was slightly lower compared to frozen tissues regarding TERTp mutations $(37.0 \%$ vs. $42.0 \%$, respectively). This may be because the use of FFPE tissue samples could give rise to high falsenegative rates, and a large proportion of normal tissue reduced the positive rate of mutant cancer cells [37]. In a recent study, it was revealed that formalin fixation might result in the deamination of cytosine residues, DNA degradation, and single-nucleotide variants [38, 39]. However, FFPE samples are suitable for long-term storage and can be widely used for molecular detection [40]. Therefore, our Sanger sequencing for TERTp mutations can be applied to routinely process frozen tissue or FFPE tissue and provided the required DNA quality and tumor purity.

We additionally investigated the relationship between three biomarkers (TERTp, IDH1/2, and MGMT methylation status) and clinicopathological features. In line with recent studies [41], the frequency of TERTp mutations correlated with an older age. TERTp mutations could accelerate cancer cell proliferation and DNA repair [42]. Taken together, these findings may explain the fact that TERTp mutations could be one of the main causes in elderly patients. On the contrary, IDH mutations were predominantly found in younger patients, at a frontal location, and in pathologic stage II-III patients, which agreed with prior studies [42, 43]. Moreover, in the context of our findings, IDH mutations were significantly associated with MGMT promoter methylation. Prior studies have shown that patients harboring IDH mutations have an improved outcome when compared with IDH wild-type patients [44]. Lu et al. demonstrated that IDH mutations changed the enzyme substrate affinity, leading to the methylation of cytosine-phosphate-guanine islands, including the MGMT promoter [45].

$\mathrm{Ki}-67$ protein expression is associated with cell cycle and accelerates tumor progression by promoting cell proliferation and metastasis [46]. Our findings indicated that Ki-67 protein expression was lower in IDH mutation tumors and was the highest in the IDH-wt/TERTp-mut group, thereby indicating that IDH-wt and TERTp-mut have a greater 
capacity to promote tumor growth and progression. Data by Coons et al. supported the view that Ki-67 protein expression was related to the proliferative activity and outcome of oligodendrogliomas [47].

In our study, we successfully established a Sanger sequencing-based molecular screening method for detecting TERT promoter mutations in glioma samples. Compared to traditional sequencing, this method is more rapid, convenient, and simple. We found that glioma cell line U251 harbored the C228T TERTp homozygous mutation and helped us with performance verification. Moreover, we completed the association of both TERTp mutations and IDH mutations with MGMT methylation and Ki-67 protein expression. Because the study contains a limited number of samples, investigating the distribution frequency of the abovementioned four groups in each histological type could not be performed. However, this lack of data will not affect a clinician's decisions about a patient's treatment. In summary, a simple and accurate method for screening of TERTp mutations may aid in and earlier diagnosis and prognosis evaluation of gliomas.

\section{Data Availability}

All data generated or analyzed during this study are included in this published article.

\section{Conflicts of Interest}

The authors declare that they have no conflicts of interest.

\section{Authors' Contributions}

SJB and XSL designed the study. YLZ and YL performed the experiments. FH analyzed the data. HLB and WC wrote this manuscript. All authors read and approved the final manuscript. Huili Bai contributed equally to this work.

\section{Acknowledgments}

We would like to thank Dr. Peng Xie (Chongqing Key Laboratory of Neurobiology, Chongqing Medical University) for the gift of human glioma cell lines U251 and OL. This study was supported by the National Natural Science Foundation of China (grant no. 81871653) and Chongqing Medical Science Project (grant no. 2018MSXM065).

\section{Supplementary Materials}

Figure S1: representative examples of TERTp mutations by Sanger sequencing in glioma patients. (A) wild-type. (B) TERTp C250T heterozygous mutation. (C) TERTp C228T heterozygous mutation. Figure S2: representative HE stains of frozen and FFPE tissues in glioma samples. (A) frozen tissues. (B) FFPE tissues. The HE stains images were taken at $\times 400$ magnification. Scale bars represent $100 \mu \mathrm{m}$. Figure S3: representative examples of IDH1 mutations by the Sanger sequencing and Ki-67 stains by immunohistochemistry. (A) IDH1 wild-type. (B) IDH1 R132H mutation (the nucleotide changes of G395A). (C) Ki-67 stains negative, Ki-67 < 15\%.
(D) Ki-67 stains positive, Ki-67 $\geq 15 \%$. The Ki-67 stains images were taken at $\times 400$ magnification. Scale bars represent $100 \mu \mathrm{m}$. Figure S4: MSP of MGMT promoter status in glioma samples. Unmethylation samples showed only unmethylated band with no methylated MGMT, while methylated and unmethylated bands were found in MGMT methylation samples. MSP: Methylation-specific PCR; M: PCR product amplified by methylated-specific primers; U: PCR product amplified by unmethylated-specific primers; P: positive sample; N: negative sample; B: blank control. Table SI: comparison of TERTp mutations detected by the Sanger sequencing and RT-PCR method in glioma patients. (Supplementary Materials)

\section{References}

[1] A. Behin, K. Hoang-Xuan, A. F. Carpentier, and J. Y. Delattre, "Primary brain tumours in adults," Lancet, vol. 361, no. 9354, pp. 323-331, 2003.

[2] Y. Yan, Z. Xu, X. Chen et al., "Novel function of lncRNA ADAMTS9-AS2 in promoting temozolomide resistance in glioblastoma via upregulating the FUS/MDM2 ubiquitination axis," Frontiers in Cell and Development Biology, vol. 7, p. 217, 2019.

[3] X. Chen, Z. Xu, S. Zeng et al., "SIRT5 downregulation is associated with poor prognosis in glioblastoma," Cancer Biomarkers: A Disease Markers, vol. 24, no. 4, pp. 449-459, 2019.

[4] Z. Xu, X. Wang, X. Chen et al., "Identification of aloperine as an anti-apoptotic Bcl2 protein inhibitor in glioma cells," PeerJ, vol. 7, article e7652, 2019.

[5] Q. T. Ostrom, G. Cioffi, H. Gittleman et al., "CBTRUS statistical report: primary brain and other central nervous system tumors diagnosed in the United States in 2012-2016," NeuroOncology, vol. 21, Supplement_5, pp. v1-v100, 2019.

[6] M. J. van den Bent, "Interobserver variation of the histopathological diagnosis in clinical trials on glioma: a clinician's perspective," Acta Neuropathologica, vol. 120, no. 3, pp. 297304, 2010.

[7] X. Li, Q. Tang, J. Yu, Y. Wang, and Z. Shi, "Microvascularity detection and quantification in glioma: a novel deeplearning-based framework," Laboratory Investigation, vol. 99, no. 10, pp. 1515-1526, 2019.

[8] X. Chen, Y. Yan, J. Zhou et al., "Clinical prognostic value of isocitrate dehydrogenase mutation, O-6-methylguanineDNA methyltransferase promoter methylation, and $1 \mathrm{p} 19 \mathrm{q}$ co-deletion in glioma patients," Annals of translational Medicine, vol. 7, no. 20, p. 541, 2019.

[9] M. B. S. Lopes, “The 2017 World Health Organization classification of tumors of the pituitary gland: a summary," Acta Neuropathologica, vol. 134, no. 4, pp. 521-535, 2017.

[10] F. W. Huang, E. Hodis, M. J. Xu, G. V. Kryukov, L. Chin, and L. A. Garraway, "Highly recurrent TERT promoter mutations in human melanoma," Science, vol. 339, no. 6122, pp. 957-959, 2013.

[11] D. S. Huang, Z. Wang, X. J. He et al., "Recurrent TERT promoter mutations identified in a large-scale study of multiple tumour types are associated with increased TERT expression and telomerase activation," European Journal of Cancer, vol. 51, no. 8, pp. 969-976, 2015.

[12] P. S. Rachakonda, I. Hosen, P. J. de Verdier et al., “TERT promoter mutations in bladder cancer affect patient survival and 
disease recurrence through modification by a common polymorphism," Proceedings of the National Academy of Sciences of the United States of America, vol. 110, no. 43, pp. 1742617431, 2013.

[13] S. E. Lee, S. H. Chang, W. Y. Kim et al., "Frequent somatic TERT promoter mutations and CTNNB1 mutations in hepatocellular carcinoma," Oncotarget, vol. 7, no. 43, pp. 6926769275, 2016

[14] S. K. Yoo, Y. S. Song, E. K. Lee et al., "Integrative analysis of genomic and transcriptomic characteristics associated with progression of aggressive thyroid cancer," Nature Communications, vol. 10, no. 1, p. 2764, 2019.

[15] P. Subedi, S. Nembrini, Q. An et al., "Telomere length and cancer mortality in American Indians: the Strong Heart Study," GeroScience, vol. 41, no. 3, pp. 351-361, 2019.

[16] R. Santin-Marquez, A. Alarcon-Aguilar, N. E. Lopez-Diazguerrero, N. Chondrogianni, and M. Konigsberg, "Sulforaphane - role in aging and neurodegeneration," GeroScience, vol. 41, no. 5, pp. 655-670, 2019.

[17] S. Shete, F. J. Hosking, L. B. Robertson et al., "Genome-wide association study identifies five susceptibility loci for glioma," Nature Genetics, vol. 41, no. 8, pp. 899-904, 2009.

[18] F. Cheng and D. Guo, "MET in glioma: signaling pathways and targeted therapies," Journal of Experimental \& Clinical Cancer Research, vol. 38, no. 1, p. 270, 2019.

[19] D. N. Louis, A. Perry, G. Reifenberger et al., "The 2016 World Health Organization classification of tumors of the central nervous system: a summary," Acta Neuropathologica, vol. 131, no. 6, pp. 803-820, 2016.

[20] T. B. Jin, J. Y. Zhang, G. Li et al., "RTEL1 and TERT polymorphisms are associated with astrocytoma risk in the Chinese Han population," Tumour Biology, vol. 34, no. 6, pp. 36593666, 2013.

[21] L. Yang, Z. Xu, L. Liu et al., "Targeting EBV-LMP1 DNAzyme enhances radiosensitivity of nasopharyngeal carcinoma cells by inhibiting telomerase activity," Cancer Biology \& Therapy, vol. 15, no. 1, pp. 61-68, 2014.

[22] P. J. Killela, Z. J. Reitman, Y. Jiao et al., "TERT promoter mutations occur frequently in gliomas and a subset of tumors derived from cells with low rates of self-renewal," Proceedings of the National Academy of Sciences of the United States of America, vol. 110, no. 15, pp. 6021-6026, 2013.

[23] P. Zhao, C. Wang, Z. Fu et al., "Lentiviral vector mediated siRNA knock-down of hTERT results in diminished capacity in invasiveness and in vivo growth of human glioma cells in a telomere length-independent manner," International Journal of Oncology, vol. 31, no. 2, pp. 361-368, 2007.

[24] P. van Loo, S. H. Nordgard, O. C. Lingjaerde et al., "Allele-specific copy number analysis of tumors," Proceedings of the National Academy of Sciences of the United States of America, vol. 107, no. 39, pp. 16910-16915, 2010.

[25] J. K. Myung, S. J. Byeon, B. Kim et al., "Papillary glioneuronal tumors," The American Journal of Surgical Pathology, vol. 35, no. 12, pp. 1794-1805, 2011.

[26] X. Rao, X. Huang, Z. Zhou, and X. Lin, “An improvement of the $2^{\wedge}$ (-delta delta CT) method for quantitative real-time polymerase chain reaction data analysis," Biostatistics, Bioinformatics and Biomathematics, vol. 3, no. 3, pp. 71-85, 2013.

[27] S. S. Menon, C. Guruvayoorappan, K. M. Sakthivel, and R. R. Rasmi, "Ki-67 protein as a tumour proliferation marker," Clinica Chimica Acta, vol. 491, pp. 39-45, 2019.
[28] B. H. Diplas, H. Liu, R. Yang et al., "Sensitive and rapid detection of TERT promoter and IDH mutations in diffuse gliomas," Neuro-Oncology, vol. 21, no. 4, pp. 440-450, 2019.

[29] J. Liu, Z. Zhao, M. Sun, K. Chen, W. Yuan, and G. Jiang, "The sensitive detection of telomerase reverse transcriptase promoter mutation by Amplification Refractory Mutation System-PCR," Genetic Testing and Molecular Biomarkers, vol. 20, no. 2, pp. 90-93, 2016.

[30] L. Qin, W. Zhong, L. Zhang, L. Y. Li, and M. Z. Wang, "Comparison of three methods for detecting epidermal growth factor receptor mutations in plasma DNA samples of Chinese patients with advanced non-small cell lung cancer," Chinese Medical Journal, vol. 124, no. 6, pp. 887-891, 2011.

[31] G. R. Oxnard, C. P. Paweletz, Y. Kuang et al., "Noninvasive detection of response and resistance in EGFR-mutant lung cancer using quantitative next-generation genotyping of cellfree plasma DNA," Clinical Cancer Research, vol. 20, no. 6, pp. 1698-1705, 2014.

[32] S. A. Stewart, W. C. Hahn, B. F. O'Connor et al., "Telomerase contributes to tumorigenesis by a telomere lengthindependent mechanism," Proceedings of the National Academy of Sciences of the United States of America, vol. 99, no. 20, pp. 12606-12611, 2002.

[33] A. Catteau, H. Girardi, F. Monville et al., "A new sensitive PCR assay for one-step detection of 12 IDH1/2 mutations in glioma," Acta Neuropathologica Communications, vol. 2, no. 1, p. 58, 2014.

[34] P. Yang, J. Cai, W. Yan et al., "Classification based on mutations of TERT promoter and IDH characterizes subtypes in grade II/III gliomas," Neuro-Oncology, vol. 18, no. 8, pp. 1099-1108, 2016.

[35] C. Koelsche, F. Sahm, D. Capper et al., "Distribution of TERT promoter mutations in pediatric and adult tumors of the nervous system," Acta Neuropathologica, vol. 126, no. 6, pp. $907-$ 915, 2013.

[36] H. Arita, Y. Narita, S. Fukushima et al., "Upregulating mutations in the TERT promoter commonly occur in adult malignant gliomas and are strongly associated with total $1 \mathrm{p} 19 \mathrm{q}$ loss," Acta Neuropathologica, vol. 126, no. 2, pp. 267-276, 2013.

[37] M. J. Kwon, J. Y. Jeon, H. R. Park et al., "Low frequency of KRAS mutation in pancreatic ductal adenocarcinomas in Korean patients and its prognostic value," Pancreas, vol. 44, no. 3, pp. 484-492, 2015.

[38] L. M. Prentice, R. R. Miller, J. Knaggs et al., "Formalin fixation increases deamination mutation signature but should not lead to false positive mutations in clinical practice," PLoS One, vol. 13, no. 4, article e0196434, 2018.

[39] S. Kim, C. Park, Y. Ji et al., "Deamination effects in formalinfixed, paraffin-embedded tissue samples in the era of precision medicine," The Journal of Molecular Diagnostics, vol. 19, no. 1, pp. 137-146, 2017.

[40] R. M. Ferreira, J. Pereira-Marques, I. Pinto-Ribeiro et al., "Gastric microbial community profiling reveals a dysbiotic cancer-associated microbiota," Gut, vol. 67, no. 2, pp. 226 236, 2018.

[41] J. E. Eckel-Passow, D. H. Lachance, A. M. Molinaro et al., "Glioma groups based on 1p/19q, IDH, and TERT promoter mutations in tumors," The New England Journal of Medicine, vol. 372, no. 26, pp. 2499-2508, 2015. 
[42] H. Yan, D. W. Parsons, G. Jin et al., "IDH1 and IDH2 mutations in gliomas," The New England Journal of Medicine, vol. 360, no. 8, pp. 765-773, 2009.

[43] M. Weller, J. Felsberg, C. Hartmann et al., "Molecular predictors of progression-free and overall survival in patients with newly diagnosed glioblastoma: a prospective translational study of the German Glioma Network," Journal of Clinical Oncology, vol. 27, no. 34, pp. 5743-5750, 2009.

[44] M. J. van den Bent, M. Weller, P. Y. Wen, J. M. Kros, K. Aldape, and S. Chang, "A clinical perspective on the 2016 WHO brain tumor classification and routine molecular diagnostics," Neuro-Oncology, vol. 19, no. 5, pp. 614-624, 2017.

[45] C. Lu, P. S. Ward, G. S. Kapoor et al., "IDH mutation impairs histone demethylation and results in a block to cell differentiation," Nature, vol. 483, no. 7390, pp. 474-478, 2012.

[46] A. Pollack, M. DeSilvio, L. Y. Khor et al., "Ki-67 staining is a strong predictor of distant metastasis and mortality for men with prostate cancer treated with radiotherapy plus androgen deprivation: Radiation Therapy Oncology Group Trial 9202," Journal of Clinical Oncology, vol. 22, no. 11, pp. 21332140, 2004.

[47] S. W. Coons, P. C. Johnson, and D. K. Pearl, "The prognostic significance of Ki-67 labeling indices for oligodendrogliomas," Neurosurgery, vol. 41, no. 4, pp. 878-885, 1997. 